\title{
Independent Model Generalized Predictive Controller Design for Antilock Braking System
}

\author{
M. Hasan Khansari \\ Department of Electrical \\ Engineering \\ Khomeini Shahr Branch, \\ Islamic Azad University \\ Isfahan, Iran
}

\author{
Mahdi Yaghoobi \\ Department of Electrical \\ Engineering \\ Mashhad Branch, Islamic Azad \\ University \\ Mashhad, Iran
}

\author{
Alireza Abaspour \\ Space Research Institute \\ Maleke_Ashtar University of \\ Technology \\ Tehran, Iran
}

\begin{abstract}
Wheel slip control is a significant research topics in the field of car stability. Model predictive control is one of the most advanced controller which has received great attention and application in industries. In this paper independent model generalize predictive control (IMGPC) is introduced for antilock braking system. This controller is implemented on a linear model of anti-lock braking system, and through the numerical simulation, it is demonstrated that it can control the system in presence of sever noise and disturbances. The simulation results show that the proposed controller has better performance in comparison with other conventional linear controllers.
\end{abstract}

\section{General Terms}

Predictive control, Anti-lock braking system .

\section{Keywords}

Anti-lock braking system, generalized predictive control, ABS linearized model, robustness

\section{INTRODUCTION}

Anti-lock braking system (ABS) is made to prevent car wheels from slip and subsequent instability in sudden braking situation. The changes in friction, vehicle mass, road inclination and other nonlinearities in dynamic can influence the ABS performance. These kind of systems, which has highly nonlinear properties, cannot be accurately controlled by classical control strategies. Therefore, this paper investigates control strategies which can be applied on ABS control system.

Conventional control approaches may not be sufficient for the aforementioned challenges. This is because of the necessity in accurate control of these kind of systems. For an accurate control design, it is essential to obtain an accurate dynamic model which is appropriate for the controller. Various control techniques has been applied for the control of the ABS, and subsequently various kind of dynamic model have been developed. Poursamad et. al introduce nonlinear model of the ABS which is applicable for nonlinear control strategies such as feedback linearization control [1]. Feedback linearization control is a nonlinear control strategy which have been applied for various kind of systems [1-3]. Dousti et al introduced a multiple model switching design for ABS control [4]. This multiple switching model was based on observer prediction signals according to its preset wheel model, and led to enhance adaption in presence of changing in driving and road conditions. Radu et al. presented a particle swarm optimization (PSO) for tuning the fuzzy controller which was designed for a linear dynamic model of ABS [5]. Johansen et. al Introduced a gain scheduling linear quadratic regulator
(LQR) controller for ABS [6]. They have also implement this controller on actual car system, and obtained efficient control performances. Raesian et. al introduced a model predictive control design for antilock braking system in which the Imperialist competitive algorithm (ICA) was used for quadratic programming $(\mathrm{QP})$ minimization which occurs in the calculation of the model predictive control (MPC) at each sampling interval [7]. Anwar et. al presented Generalized predictive control based on 'Controlled Auto Regressive Integrated Moving Average (CARIMA)' model for ABS system [8]. This controller is was tested in normal condition, and without any noise and disturbances.

According to the progress in control methodologies in recent years, predictive control techniques have received much of attention among the control designers, and it is predicted that based on its control advantages and progress in computer calculation abilities, its application in industries will be more broaden than now [9]. Generalized predictive control (GPC) strategy is a discrete control procedure which is introduced by Clarke et. al $[10,11]$. In this control strategy, unlike the dynamic matrix control (DMC) and model algorithm control (MAC), the system model does not need to be stable, because predicted output of process is only predicted based on predicted system model. Thus, with prediction of the future system output, the control process is done by considering future output error.

Recent researches [12,13] showed that GPC controllers cannot tolerate severe noise and disturbances, and it's performance may degrade in presence of them. Moreover, accurate GPC control procedure needs a special transfer function model, which is named CARIMA. Obtaining this CARIMA model is usually difficult, and needs complex calculations [14]. Furthermore, in multi input multi output systems (MIMO), GPC controller needs an observer for state estimation. For these reasons, the independent model generalized predictive (IMGPC) controller is introduced by Rossiter [13].

In this research, IMGPC technique is applied for antilock braking system control. The control system is tested under sever disturbances and noises. The numerical simulation results show that the proposed controller can efficiently control the ABS braking system. The controller robustness was tested in presence of severe noise and disturbances, and the simulation results show that its noise and disturbance rejection performance is quite better in comparison with $L Q R$ controller.

The paper is organized as follows: in section 2 the mathematical model of ABS is extracted, GPC and the IMGPC technique is described in section 3. Then in section 4 the numerical simulation is done, while the conclusions are provided in section 5 . 


\section{DYNAMIC MODEL}

In this section, we present a mathematical dynamic model of wheel slip. The problem of wheel slip control is best illustrated by a quarter car model as depicted on Figure 1. The model consists of a single wheel which is attached to a mass ( $m$ ). As the wheel rotates in the direction of the velocity $(v)$ by the inertia of the mass $(m)$, the friction between the tire and surface will led to a tire reaction force $\left(F_{x}\right)$. This tire reaction force will generate a torque that leads to a rolling motion of the wheel which will cause an angular velocity ( $\omega$ ). The control input in this system which can be applied to the wheel is a brake torque $\left(T_{b}\right)$, and it will act against the wheel spinning and causing a negative angular acceleration. The aforementioned system is described as follow:

$m \dot{v}=-F_{x}$

$J \dot{\omega}=r F_{x}-T_{b} \operatorname{sign}(\omega)$

where $v$ is longitudinal speed of vehicle ; $\omega$ is the wheel angular speed; $T_{b}$ is brake torque; $F_{z}$ is vertical force; $F_{x}$ tire friction force; $r$ is wheel radius; and $J$ is wheel inertia.

The equation of tire friction force is described by:

$F_{x}=F_{z} \cdot \mu\left(\lambda, \mu_{H}, \alpha\right)$

$\mu$ is the friction coefficient which is a nonlinear function of $\lambda$, and $\lambda$ is the longitudinal tire slip. $\mu_{H}$ is the maximum friction coefficient between tire and road, and $\alpha$ is the wheel slip angle .

The longitudinal slip can be defined as follow :

$\lambda=\frac{v-\omega r}{v}$

$\lambda=0$ means that there is no friction force $F_{x}$ and wheel has free motion. If the slip reaches the value of $\lambda=1$, then the wheel is locked $(\omega=0)$. The friction coefficient value can be varied in different situation. The relation between the friction and slip on the road condition is shown in the Figure 2. As it can be seen for icy or wet roads, the maximum friction $\mu_{H}$ is smaller. If we consider the lateral motion of the wheel, the wheel motion will be extended to two direction. The slip angle $\alpha$ is the angle between the longitudinal speed $\left(v_{x}\right)$ and the lateral speed $\left(v_{y}\right)$. In this research, the longitudinal sleep is $\lambda_{x}=\frac{v_{x}-\omega r}{v}$ and the lateral slip is $\lambda_{y}=\sin \alpha$, and subsequently, the corresponding friction coefficient considered as $\mu_{x}$, and $\mu_{y}$. The dependence of friction coefficient $\mu$ and the slip angle is depicted on the top part of Figure 2. In this paper for simplification purposes, the slip angle is considered to be zero, therefore, $\mu_{x}=\mu$ and $v_{x}=0$.

Considering $v>0$ and $\omega>0$ and (1)-(4) :

$$
\begin{aligned}
& \dot{\lambda}=-\frac{1}{v}\left(\frac{1}{m}(1-\lambda)+\frac{r^{2}}{J}\right) F_{z} \mu\left(\lambda, \mu_{H}, \alpha\right)+\frac{1}{v} \frac{r}{J} T_{b} \\
& \dot{v}=-\frac{1}{m} F_{z} \mu\left(\lambda, \mu_{H}, \alpha\right)
\end{aligned}
$$

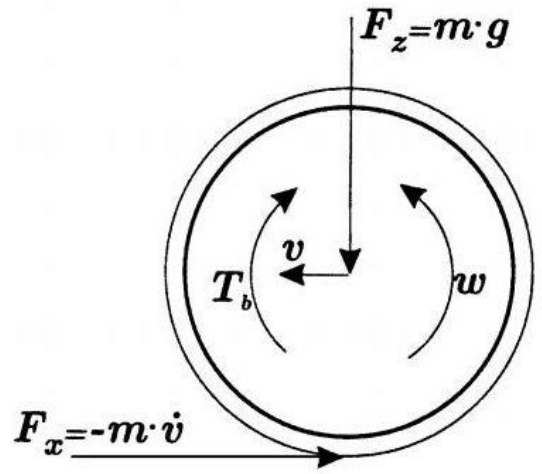

Fig. 1: Wheel dynamic model
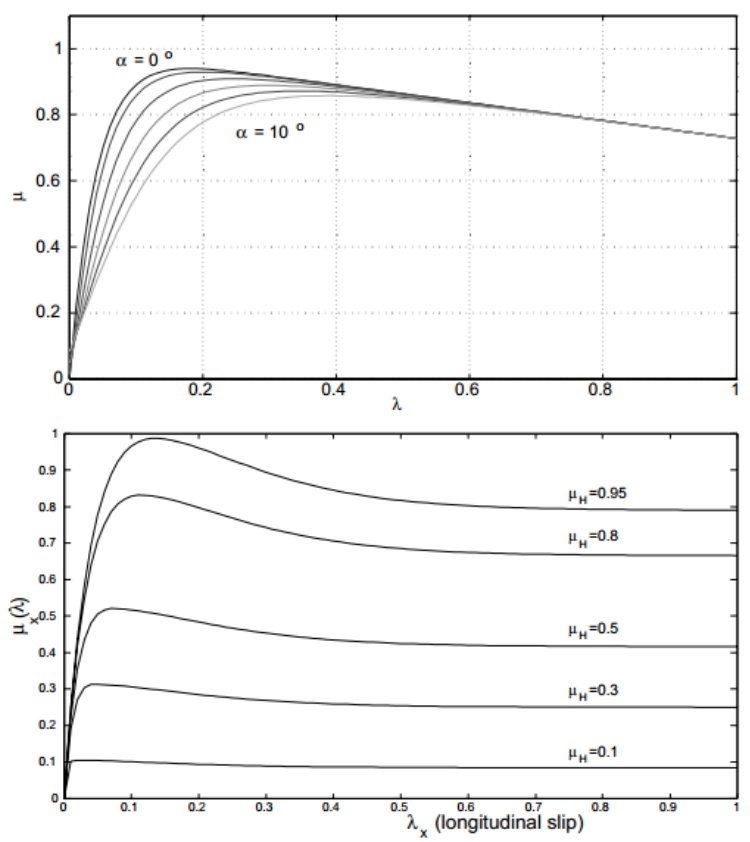

Fig. 2: Slip/friction curves of Tyre [7]

\subsection{Linearized Dynamic}

The GPC and IMGPC controller which is discussed in this paper is based on linearized dynamic; therefore, the aforementioned nonlinear equation of wheel slip model should be linearized.

By assuming $\left(\lambda^{*}, \hat{T}_{b}\right)$ as an equilibrium for (5), we have:

$\hat{T}_{b}=\left(\frac{J}{m r}\left(1-\lambda^{*}\right)+r\right) F_{z}^{*} \mu\left(\lambda^{*}, \mu_{H}^{*}, \alpha^{*}\right)$

The linearized slip dynamic are equivalent by:

$\dot{\lambda}=\frac{\alpha_{1}}{v}\left(\lambda-\lambda^{*}\right)+\frac{\beta_{1}}{v}\left(T_{b}-\hat{T}_{b}\right)+$ h.o.t . 
Where $\alpha_{1}$ and $\beta_{1}$ are the linearization constant which are given by:

$\alpha_{1}=-F_{z}^{*}\left(\frac{1}{m}\left(1-\lambda^{*}\right)+\frac{r^{2}}{J}\right) \frac{\partial \mu}{\partial \lambda}\left(\lambda^{*}, \mu^{*}, \alpha\right)+$

$F_{z}^{*} \frac{1}{m} \mu\left(\lambda^{*}, \mu_{H}^{*}, \alpha^{*}\right)$

$\beta_{1}=\frac{r}{J}>0$

Considering (7-10) and $x_{2}=\lambda-\lambda^{*}$, the wheel slip dynamics can be rewritten as follow:

$\dot{x}_{2}=\frac{\phi\left(x_{2}\right)}{v}+\frac{\beta_{1}}{v}\left(T_{b}-T_{b}^{*}\right)$

Where

$\phi\left(x_{2}\right)=-\left(\frac{1}{m}\left(1-\lambda^{*}-x_{2}\right)+\frac{r^{2}}{J}\right) \times$

$F_{z} \mu\left(x_{2}+\lambda^{*}, \mu_{H}, \alpha\right)+\frac{r}{J} T_{b}^{*}$

$T_{b}^{*}=\left(\frac{J}{m r}\left(1-\lambda^{*}\right)+r\right) F_{z} \mu\left(\lambda^{*}, \mu_{H}, \alpha\right)$

It can be seen that the above system has an equilibrium point in $x_{2}=0, T_{b}=T_{b}^{*}$ and $\phi(0)=0$ and the linearized slip model (8) with perturbation term written as follows

$\dot{x}_{2}=\frac{\alpha_{1}}{v} x_{2}+\frac{\beta_{1}}{v}\left(T_{b}-T_{b}^{*}\right)+\frac{\in_{\mu}\left(x_{2}\right)}{v}$

where $\epsilon_{\mu}\left(x_{2}\right)=\phi\left(x_{2}\right)-\alpha_{1} x_{2}$.

By augmenting the system dynamic with a slip error integrator $\dot{x}_{1}=\lambda-\lambda^{*}=x_{2}$, we have:

$\left(\begin{array}{l}\dot{x}_{1} \\ \dot{x}_{2}\end{array}\right)=A(v)\left(\begin{array}{l}x_{1} \\ x_{2}\end{array}\right)+B(v)\left(u-T_{b}^{*}\right)+W(v) \in_{\mu}\left(x_{2}\right)$

Where

$A(v)=\left(\begin{array}{cc}0 & 1 \\ 0 & \frac{\alpha_{1}}{v}\end{array}\right), B(v)=\left(\begin{array}{c}0 \\ \frac{\beta_{1}}{v}\end{array}\right), W(v)=\left(\begin{array}{c}0 \\ \frac{1}{v}\end{array}\right)$

In this paper we also considered the electromechanical actuator dynamics, which is sufficiently approximated the following first order discrete-time linear model $(7 \mathrm{~ms}$ sampling interval):

$T_{b}(t+1)=a_{a c t} T_{b}(t)+b_{a c t} \tilde{T}_{b}(t)$

Where $a_{a c t}=0.6$ and $b_{a c t}=0.4$ corresponding to an actuator bandwidth of $72 \mathrm{rad} / \mathrm{s}$. $T_{b}$ is the brake torque and
$\tilde{T}_{b}$ is the brake torque which is commanded to the brake actuator servo.

In order to increase the accuracy of dynamic model, the second-order linearized model (15) is discretize and augment with an actuator model. In addition, the changes in commanded brake torque is considered as the control input, therefore, this can simplifies the handling of actuator rate constraints in the controller. Furthermore, this converts the model in a velocity form that is favorable for predictive control design [14]. Consequently, we obtain a fourth-order discrete-time linear parameter-varying (LPV) state space model:

$$
x(t+1)=A(v) x(t)+B u(t)
$$

where $x_{1}$ is the integrated slip error, $x_{2}=\lambda-\lambda^{*}$ is the slip error, $x_{3}=T_{b}$ is the actuator brake and $x_{4}=\tilde{T}_{b}$ is the brake torque which is commanded to the actuator. The speeddependent model matrices are described by:

$$
A(v)=\left[\begin{array}{cccc}
1 & T_{s} & 0 & 0 \\
0 & a_{1}(v) & b_{1}(v) & 0 \\
0 & 0 & a_{a c t} & b_{a c t} \\
0 & 0 & 0 & 1
\end{array}\right] ; B=\left[\begin{array}{l}
0 \\
0 \\
0 \\
1
\end{array}\right]
$$

Where the discretization parameters are defined as follows:

$$
a_{1}(v)=e^{T_{s} \alpha_{1} / v}, \quad b_{1}(v)=\beta_{1}\left(a_{1}(v)-1\right) \alpha_{1}
$$

\section{IMGPC CONTROL DESIGN}

In this section the model prediction in state -space form and also IMGPC control design strategy are described. This paper introduces IMGPC method as an alternative for GPC technique. As it mentioned in the introduction part, GPC controller design is based on CARIMA model, which obtaining an accurate CARIMA model for MIMO system is quite difficult. Therefore, this paper presents IMGPC technique controller for designing ABS controller. The IMGPC method which is introduced in this paper is based on the state-space model; thus, the control designer is more convenient in designing controller in comparison with GPC method.

\subsection{Model Predicting in State-Space Form}

The state-space model can be described by following equation:

$$
\begin{aligned}
& x_{k+1}=A x_{k}+B u_{k} \\
& y_{k}=C x_{k}+D u_{k}+d_{k}
\end{aligned}
$$

Where $x_{k}$ is the state-space variable, $u_{k}$ is the control input, and $y_{k}$ is the system output. For simplification, the $D$ coefficient is considered to be zero. Therefore, we have:

$$
y_{k+1}=C A x_{k}+C B u_{k}+d_{k}
$$

The model prediction step can be describe by following equation: 


$$
\left.\begin{array}{l}
x_{k+1}=A x_{k}+B u_{k} \\
x_{k+2}=A x_{k+1}+B u_{k+1} \\
x_{k+3}=A x_{k+2}+B u_{k+2} \\
x_{k+4}=A x_{k+3}+B u_{k+3}
\end{array}\right\} \Rightarrow
$$

As it can be seen in (23), there is a relation among each step of prediction. By multiplying the above parameters, the simplified form of (23) can be described as follow:

$$
\begin{aligned}
& x_{k+1}=A x_{k}+B u_{k} \\
& x_{k+2}=A^{2} x_{k}+A B u_{k}+B u_{k+1} \\
& x_{k+3}=A^{3} x_{k}+A^{2} B u_{k}+A B u_{k+1}+B u_{k+2} \\
& x_{k+4}=A^{4} x_{k}+A^{3} B u_{k}+A^{2} B u_{k+1}+A B u_{k+2}+B u_{k+3}
\end{aligned}
$$

The following relation can be found among the prediction steps:

$x_{k+n}=A^{n} x_{k}+A^{n-1} B u_{k}+A^{n-2} B u_{k}+\ldots$

$+A B u_{k+n-2}+B u_{k+n-1}$

By using (25), the system output can be predicted as follows:

$y_{k+n}=C x_{k+n}+d_{k+n} ; d_{k+n}=d_{k}$

$y_{k+n}=C A^{n} x_{k}+C\left(A^{n-1} B u_{k}+A^{n-2} B u_{k}+\ldots\right.$

$\left.+A B u_{k+n-2}+B u_{k+n-1}\right)+d_{k}$

The (25), can be described as a state space form as follow :

$\underline{x}_{k+1}=\left[\begin{array}{c}A x_{k} \\ A^{2} x_{k} \\ \vdots \\ A^{n} x_{k}\end{array}\right]+$

$$
\left[\begin{array}{c}
A B u_{k} \\
A B u_{k}+B u_{k+1} \\
\vdots \\
A^{n-1} B u_{k}+A^{n-2} B u_{k+1}+\ldots+A B u_{k+n-2}+B u_{k+n-1}
\end{array}\right]
$$

The (27) can be describe as a matrix form as follow :

$$
x_{k+1}=\left[\begin{array}{c}
A \\
A^{2} \\
\vdots \\
\underbrace{A^{n}}_{P_{x}}
\end{array}\right] x_{k}+
$$

$$
\left[\begin{array}{cccc}
B & 0 & \cdots & 0 \\
A B & B & \cdots & 0 \\
\vdots & \vdots & \ddots & \vdots \\
\underbrace{A^{n-1} B}_{H_{x}} & A^{n-2} B & \cdots & B
\end{array}\right]\left[\begin{array}{c}
u_{k} \\
u_{k-1} \\
\vdots \\
\underbrace{u_{k+n-1}}_{\underline{u}_{k}}
\end{array}\right]
$$

By defining $P_{x}, H_{x}$ and $u_{k}$ as it showed in (28), the (28) can be simplified in the following form:

$\underline{x}_{k+1}=P_{x} x_{k}+H_{x} u_{k}$

The output prediction follows the same procedure:

$\underline{y}_{k+1}=\left[\begin{array}{c}C A \\ C A^{2} \\ \vdots \\ \underbrace{C A^{n}}_{P}\end{array}\right] x_{k}+$

$$
\left.\begin{array}{l}
{\left[\begin{array}{cccc}
C B & 0 & \cdots & 0 \\
C A B & C B & \cdots & 0 \\
\vdots & \vdots & \ddots & \vdots \\
C A^{n-1} B & C A^{n-2} B & \cdots & C B
\end{array}\right]} \\
\Rightarrow y_{k+1}=\underbrace{P x_{k}+L d_{k}}_{\text {Past }}+\underbrace{H \underbrace{u_{k}}_{u_{k}}}_{\text {future input }}
\end{array}\right]\left[\begin{array}{c}
u_{k} \\
u_{k+1} \\
\vdots \\
u_{k+n-1}
\end{array}\right]+\left[\begin{array}{c}
d_{k} \\
d_{k} \\
\vdots \\
d_{k} \\
L d
\end{array}\right]
$$

As it can be seen on (30), we can separate the variable to two categories: Past and the future input.

\subsection{IMGPC Control Law}

The IMGPC structure is depicted on Figure 3. As it can be seen in this structure, the predicted model (Model System) and the real process (which is in presence of disturbance), are simulated at the same time. We named the difference between the real process and predicted model as offset term (d). Considering the disturbance in control design can led to accurate control design [13].

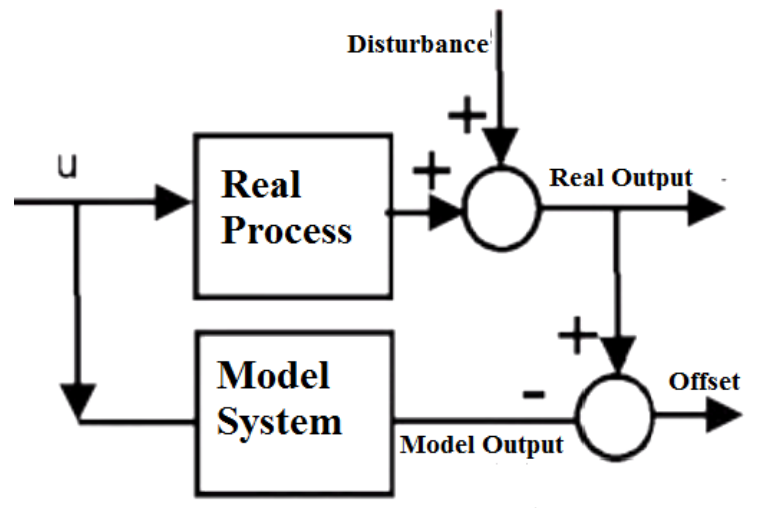

Fig. 3: The IMGPC Structure

The control law in predictive control strategies is obtained based on the minimizing the defined cost function. In this paper the cost function is defined to minimize the output error and the control input as follows:

$$
\begin{aligned}
& J=\left[\underline{r}_{k+1}^{T}-\underline{y}_{k+1}^{T}\right]\left[\underline{r}_{k+1}-\underline{y}_{k+1}\right]+ \\
& \left(\underline{u}_{k}-L u_{s s}\right) R\left(\underline{u}_{k}-L u_{s s}\right)
\end{aligned}
$$

By substituting (30) in (31), we have: 
$\left[\underline{r}_{k+1}^{T}-P_{x} x_{k}-H \underline{u}_{k}-L d_{k}\right]\left[\underline{r}_{k+1}^{T}-P_{x} x_{k}-H \underline{u}_{k}-L d_{k}\right]$

$+\left(\underline{u}_{k}-L u_{s s}\right) R\left(\underline{u}_{k}-L u_{s s}\right) \Rightarrow$

$\underbrace{\min }_{\Delta u_{k}}\left\{\begin{array}{l}\underline{u}_{k}^{T}\left(H^{T} H+R\right) \underline{u}_{k}- \\ 2 \underline{u}_{k}^{T}\left(H^{T}\left[r_{k+1}-P_{x} x_{k}-L d_{k}\right]+R L u_{s s}\right)\end{array}\right\}$

By solving the $\operatorname{grad}(J)=0$, the IMGPC control law can be obtained as follows:

$\underline{u}_{k}=\left(H^{T} H+R\right)^{-1}\left(H^{T}\left[r_{k+1}-P_{x} x_{k}-L d_{k}\right]+R L u_{s s}\right)$

It is obvious that only the first optimized decision is commanded to the system, and the other predicted commands will be optimized based on the future system output. Therefore, by defining $E_{1}^{T}=[I, 0, \ldots, 0]$, the control output can be described as follows:

$\underline{u}_{k}=E_{1}^{T}\left(H^{T} H+R\right)^{-1}\left(H^{T}\left[r_{k+1}-P_{x} x_{k}-L d_{k}\right]+R L u_{s s}\right)$

The control law in (34) can be shown in a simpler structure by defining following coefficients:

$$
\begin{aligned}
& P_{r}=E_{1}^{T}\left(H^{T} H+R\right)^{-1} H^{T} \\
& K=P_{r} P_{x} ; K_{d}=P_{r} L \\
& K_{u}=E_{1}^{T}\left(H^{T} H+R\right)^{-1} R L u_{s s}
\end{aligned}
$$

By substituting (35) in (34) the control law can be shown as follows:

$\underline{u}_{k}=P_{r} \underline{r}_{k+1}-K x_{k}-K_{d} d_{k}+K_{u} u_{s s}$

\section{NUMERICAL SIMULATION}

In this paper a new state-space model based predictive controller is introduced for ABS control system. In this section numerical simulations are done to show the advantages of proposed controller. This numerical simulations are done with Matlab software. In this simulation the weighting matrix in $\mathrm{J}(R)$ is 20 , the output horizon $\left(n_{y}\right)$ is 500 , and the input horizons $\left(n_{u}\right)$ is 40 . These variables are selected based on designer designing factors and desired performance. The aim of designing ABS control is to control the wheel slip $(\lambda)$. In this paper the desired wheel slip is 0.1 , the initial wheel slip value is 0.9 , and the initial speed of vehicle is $32 \mathrm{~m} / \mathrm{s}$.

To demonstrate the advantages of proposed method in controlling the ABS system, we compared its performance with linear quadratic regulator (LQR) controller. Moreover, the system robustness is tested over a severe noise and disturbance.

Figure 4 shows that the proposed controller can control system in a short time, and the wheel slip reached the desired value with minimum oscillation. Furthermore, the performance of introduced controller is completely better in comparison with LQR controller. Figure 5 compares the control input of the two aforementioned controllers. As it can be seen the IMGPC controller has far less control input in comparison with LQR controller. This can help brake actuator to have much longer life [15].

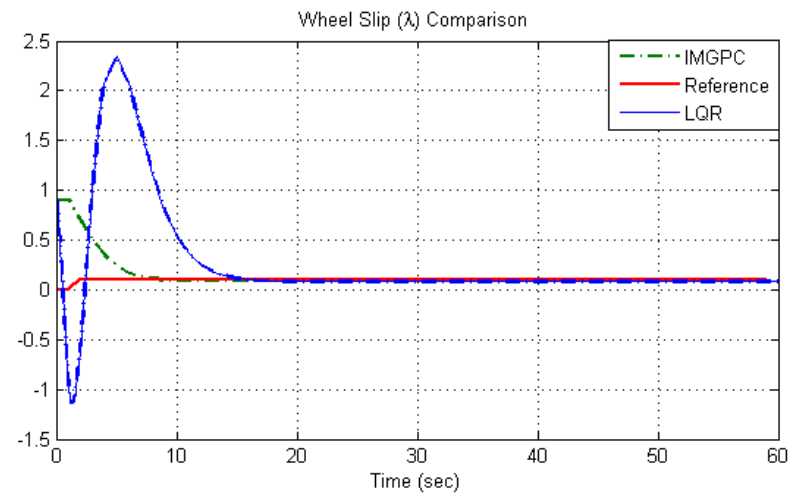

Fig. 4: Wheel slip control

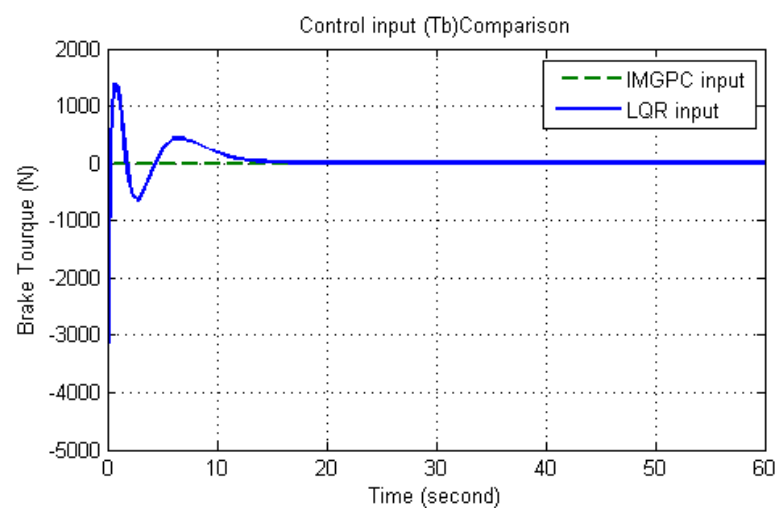

Fig. 5: Control input comparison (brake torque)

The robustness of introduced controller is tested over a sever noise and a severe disturbance which are depicted on Figure 6 . The simulation result over the noise and disturbance are depicted on Figure 7 and 8. As it can be seen in Figure 7, the IMGPC controller is quite less sensitive to noise and disturbances. In addition, Figure 8 shows that the introduced method has no chattering in presence of noise and disturbance, which is an important factor to prolong actuators life time in ABS systems.

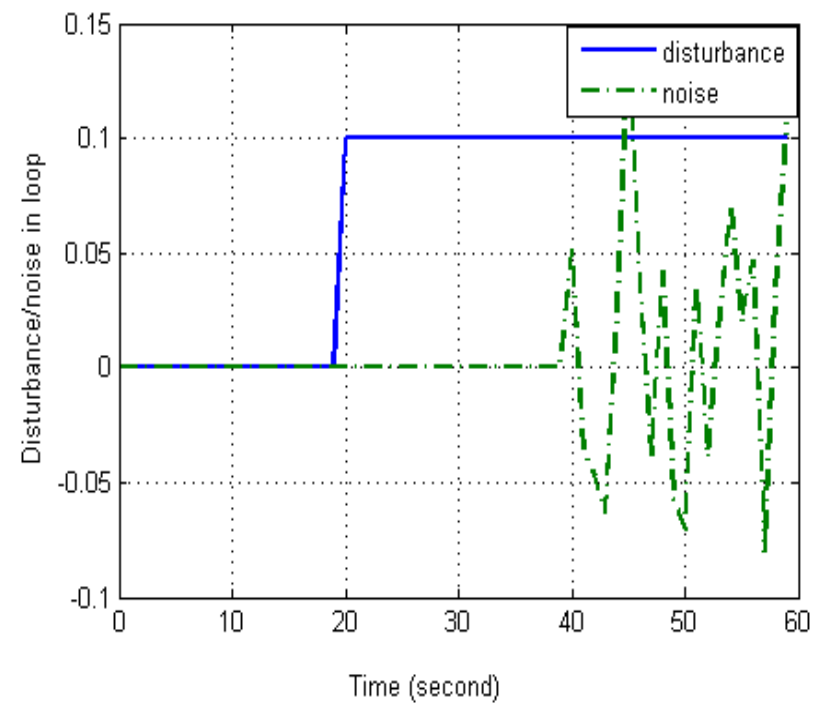

Fig. 6: Disturbance and noise in system 


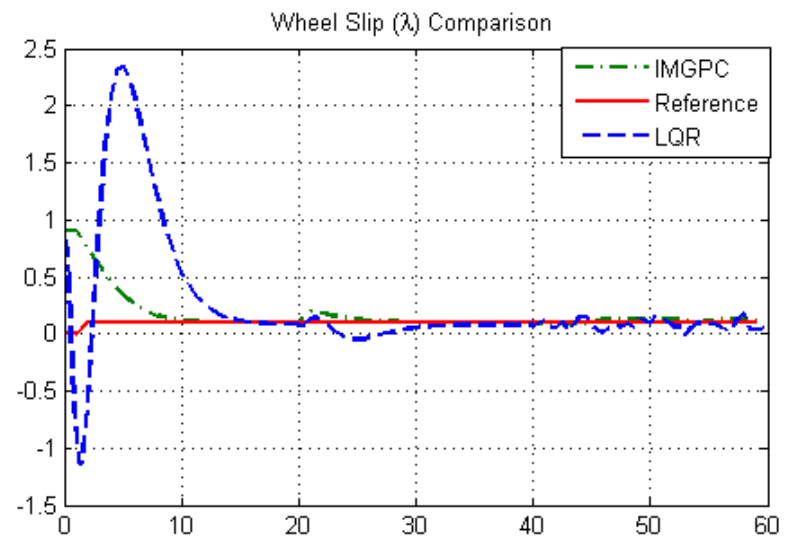

Fig. 7: Wheel slip control in presence of disturbance and noise

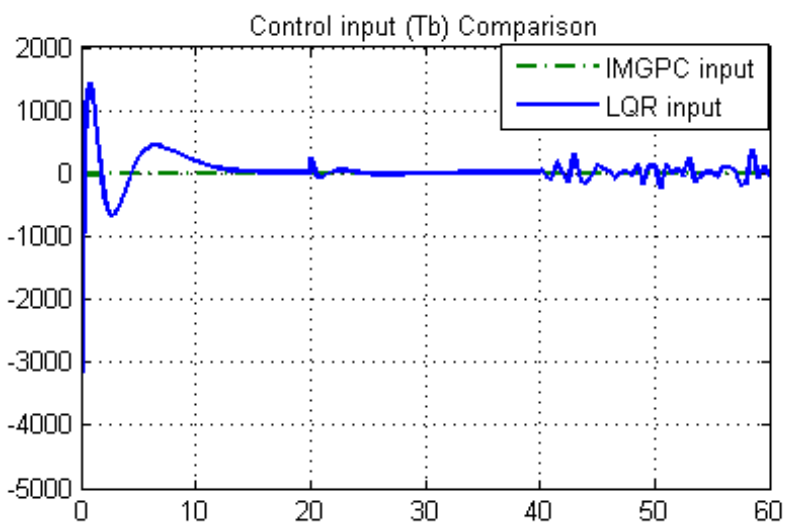

Fig. 8: Control input comparison (brake torque) in presence of noise and disturbance.

\section{CONCLUSION}

In this paper a new predictive control design for ABS control system is introduced. Generalized predictive control (GPC) is one of important control systems in industries. Designing controllers based on GPC needs CARIMA model, while obtaining CARIMA model for MIMO systems is difficult and time consuming. Therefore, this paper introduced independent model generalized predictive control (IMGPC) method which is based on state space model of system. Designing GPC controllers based on state space model can help designer to design controllers for variety of system plants. The target plant in this paper was ABS system. A speed dependent model of ABS system is obtained by linearizing the nonlinear equation of $\mathrm{ABS}$ system. By implementing the proposed controller, the simulation results show that the introduced control method can successfully control the system. Furthermore, in comparison with LQR controller, the IMGPC controller has quite better control performance. In our future work, we will try to add adaptation ability in this structure in which we believe that it can improve the control accuracy significantly.

\section{REFERENCES}

[1] Amir Poursamad, 'Adaptive feedback linearization control of antilock braking systems using neural networks', Mechatronics 19 (2009) 767-773.
[2] A. Abaspour, N.T.Parsa, M. Sadeghi, " A New Feedback Linearization-NSGA-II Based Control Design for PEM Fuel Cell" International Journal of Computer Applications, volume97/ number10/ pp.17044-7354, July 2014.

[3] A. Abaspour, M. Sadeghi, S.H. Sadati " Optimal Nonlinear Trajectory Control of an Unmanned Helicopter" The 2nd ICRoM International Conference on Robotics and Mechatronics (ICRoM 2014, Tehran, Iran on October 15-17, 2014.

[4] Morteza Dousti, S Caglar Baslamısli, E Teoman Onder, Selim Solmaz, ' Design of a multiple-model switching controller for ABS braking dynamics'. Transactions of the Institute of Measurement and Control, 1-14, 2014.

[5] Radu-Emil Precup, Marius-Csaba Sabau, Claudia-Adina Dragos, ' Particle Swarm Optimization of Fuzzy Models for Anti-lock Braking Systems', Evolving and Adaptive Intelligent Systems (EAIS), 2014 IEEE Conference on, Linz, Austria, 2-4 June 2014.

[6] A. Johansen, Idar Petersen, Jens Kalkkuhl, Jens Lüdemann, ' Gain-Scheduled Wheel Slip Control in Automotive Brake Systems ' IEEE TRANSACTIONS ON CONTROL SYSTEMS TECHNOLOGY, VOL. 11, NO. 6, NOVEMBER 2003.

[7] N.Raesian, M.Yaghoobi, M.Abedi, ' Fast Model Predictive Controller based on Imperialist Competitive Algorithm for Anti-lock Braking System(ABS) ', ICEE21, 2013.

[8] Anwar, S. and Ashrafi, B., "A Predictive Control Algorithm for an Anti-Lock Braking System," SAE Technical Paper 2002-01-0302, 2002.

[9] K. S. Holkar, L. M. Waghmare. 'An Overview of Model Predictive Control ' International Journal of Control and Automation Vol. 3 No. 4, Vol. 3 No. 4, December, 2010.

[10] Clarke, D.W., Mohtadi, C., Tuffs, P.S., 1987a. Generalized predictive control-Part I. The basic algorithm. Automatica 23, 137-148.

[11] Clarke, D.W., Mohtadi, C., Tuffs, P.S., 1987b. Generalized predictive control-Part II Extensions and interpretations. Automatica 23, 149-160.

[12] Vincent A. Akpan, George D. Hassapis, " Nonlinear model identification and adaptive model predictive control using neural networks", ISA Transactions Vol. 50, pp. 177-194, 2011

[13] J.A. Rossiter and G. Valencia-Palomo. 'Feed forward design in MPC', ECC, 2009

[14] I. Kaminer, A. M. Pascoal, P. P. Khargonekar, and E. E. Coleman, "A velocity algorithm for the implementation of gain-scheduled controllers," Automatica, vol. 31, pp. 1185-1191, 1995.

[15] A. Abaspour, M. Sadeghi, H. Sadati., "Using Fuzzy Logic In Dynamic Inversion Flight Controller With Considering Uncertainties". (2013 13th Iranian Conference on Fuzzy Systems (IFSC)). 